Possibility of applying a hydrodynamic model to describe the laser erosion of metals irradiated by high-intensity nanosecond pulses

This content has been downloaded from IOPscience. Please scroll down to see the full text. 2014 Quantum Electron. 44325

(http://iopscience.iop.org/1063-7818/44/4/325)

View the table of contents for this issue, or go to the journal homepage for more

Download details:

IP Address: 217.21.43.68

This content was downloaded on 30/06/2014 at 06:36

Please note that terms and conditions apply. 


\title{
Possibility of applying a hydrodynamic model to describe the laser erosion of metals irradiated by high-intensity nanosecond pulses
}

\author{
K.V. Kozadaev
}

\begin{abstract}
We report the results of experimental investigations of the production and development of plasma-vapour plumes upon irradiation of metal targets by nanosecond $(10-100 \mathrm{~ns})$ pulses with a high $\left(10^{8}-10^{10} \mathrm{~W} \mathrm{~cm}{ }^{-2}\right)$ power density under atmospheric conditions. The transition from a quasi-stationary thermal mechanism of metal erosion to an explosion hydrodynamic one takes place when the radiation power density increases from $10^{8}$ to $10^{9} \mathrm{~W} \mathrm{~cm}^{-2}$. The resultant experimental information is extremely important for the laser deposition of metal nanostructures under atmospheric conditions, which is possible only for power densities of $10^{8}-10^{9} \mathrm{~W} \mathrm{~cm}$.
\end{abstract}

Keywords: laser erosion of metals, nanosecond laser pulse, laser erosion plume, thermal model, hydrodynamic model.

\section{Introduction}

In accordance with the fundamental works concerned with the interaction of high-power laser radiation with materials $[1-5]$, it is customary to assume that the laser erosion of metals by pulses of moderate $\left(10^{5}-10^{8} \mathrm{~W} \mathrm{~cm}^{-2}\right)$ and high $\left(10^{8}-10^{9} \mathrm{~W} \mathrm{~cm}^{-2}\right)$ power density may be described in the framework of a thermal damage model (the so-called quasistationary ablation). According to the basic assumptions of this model, the character of metal sputtering by laser radiation is determined by the combined motion of heating, melting, and vaporisation fronts into the target, resulting in the removal of the material. Therefore, in the course of erosion there exists a sharp boundary between vapour and condensed substance, the energy transfer in the condensed phase is entirely due to electron thermal conduction, and in the gaseous phase it is entirely due to its dynamics [1]. The same model underlies more recent works concerned with the theoretical description of laser material ablation by high-intensity nanosecond pulses [6-8].

According to Ref. [1], at a laser power density $I$ above $10^{11} \mathrm{~W} \mathrm{~cm}^{-2}$ the sharp boundary between vapour and condensed substance becomes nonabrupt and turns into a macroscopic transition layer, which is in essence a very dense metal vapour. The structure and dynamics of this layer may be described by the complete system of hydrodynamic equations, whence this model of laser erosion draws its name - a hydrodynamic model. This transition layer may be compared

K.V. Kozadaev A.N. Sevchenko Research Institute of Applied Physics, Belarussian State University, ul. Kurchatova 7, 220108 Minsk, Belarus; e-mail: kozadaeff@mail.ru

Received 15 November 2013; revision received 15 January 2014 Kvantovaya Elektronika 44 (4) 325-329 (2014)

Translated by E.N. Ragozin with an explosive, which detonates to generate an overheated vapour cloud and a shock wave propagating into the target. The resultant plasma cloud, owing to its high density, is opaque to the incident laser radiation. And therefore by screening the target surface, subsequently it completely determines the character of surface-laser pulse interaction [1]. In this case, the objective of theoretical simulation of suchlike laser erosion is seriously complicated by the absence of reliable information about the absorptive properties of the macroscopic layer [6].

Uglov and Kokora [9] pointed to the 'explosive' character of laser erosion of metals even under the action of pulses with $I \sim 10^{9} \mathrm{~W} \mathrm{~cm}^{-2}$; proceeding from the hydrodynamic model, Anisimov and Luk'yanchuk [6] gave theoretical estimates for nanosecond pulses $(10-100 \mathrm{~ns})$ for $I \sim 10^{9}-10^{10} \mathrm{~W} \mathrm{~cm}^{-2}$. In this connection, the question about the limits of applicability of the thermal and hydrodynamic models for describing the laser erosion of metals by such pulses remains open.

The present work is concerned with the experimental investigation of the production and development of the vapour-plasma substance upon irradiation of metal targets by high-intensity nanosecond laser pulses. Our objective is to determine more precisely the irradiation parameters whereby there occurs a transition from the 'quasi-stationary' character of laser erosion to the 'explosive' one.

\section{Experiment}

To experimentally study the optical characteristics of laserproduced plasma (using the techniques of transverse laser probing $[10,11]$ and laser-induced plasma spectroscopy [12]) in this work we employed a research complex, which comprised the means for monitoring the dynamics of the spectral, spatial, and phase structures of laser-produced plasma plumes (LPPs) of metals with a high temporal resolution.

The research complex permits us to investigate the following optical characteristics of plasma-vapour plumes:

1) dynamics of spectrum-integrated LPP glow with a temporal resolution of $10 \mathrm{~ns}$;

2) time-integrated LPP glow spectrum with a spectral resolution of $0.12 \mathrm{~nm}$;

3) dynamics of an individual LPP spectral line intensity with spectral $(0.2 \mathrm{~nm})$ and temporal (10 ns) resolution;

4) dynamics of the intensities of the incident, transmitted, and scattered components of the probing radiation with a temporal resolution of $10 \mathrm{~ns}$;

5) dynamics of the phase composition of the plasmavapour plume (the effective diameter and density of dust phase particles) over time intervals of different duration (from $1 \mu \mathrm{s}$ to $2 \mathrm{~ms}$ ) with a temporal resolution of $10 \mathrm{~ns}$. 
The research complex is schematically represented in Fig. 1. The following basic units may be marked out in its structure: the unit for producing the driving pulse and recording its characteristics (I), the unit for investigating the spectral LPP characteristics (II), the unit for laser LPP probing (III), the unit for experimental data processing (IV), and the timing unit (V).

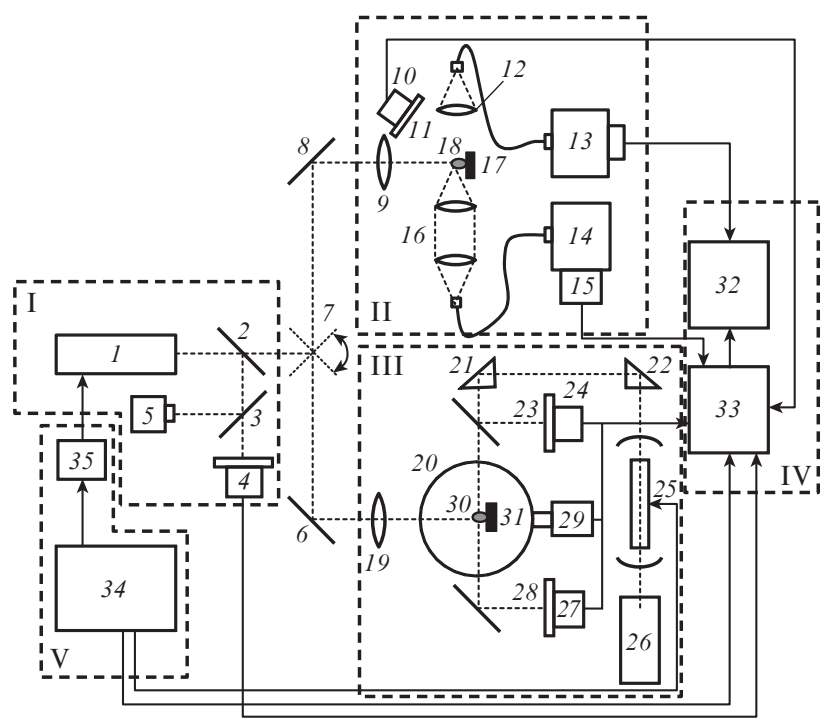

Figure 1. Schematic representation of research complex: (1) main laser; $(2,3)$ beam splitters; (4) driving-pulse shape monitor; (5) drivingpulse energy monitor; $(6-8)$ mirrors; $(9,19)$ lenses for focusing the driving radiation; (10) integral LPP glow sensor; $(11,23,28)$ optical filters; $(12,16)$ optical systems for imaging the LPP onto the entrances of spectral instruments; ( 13 ) spectrophotometer; (14) monochromator; (15, 29) PMs; $(17,31)$ target; $(18,30)$ LPP; (20) integrating sphere $(21,22)$ prisms; $(24,27)$ probe radiation intensity monitors; $(25)$ probe laser; (26) auxiliary alignment laser; (32) portable computer; (33) multichannel A to D converter; (34) clock pulse generator; (35) optical decoupler of main laser firing.

\section{Vapour-plasma substance production at atmospheric pressure}

According to classical notions, the optical radiation incident on a metal surface interacts with conduction electrons in a thin $(\sim 1$ $\mu \mathrm{m})$ surface layer, with the result that a part of radiation energy is reflected and the remaining part goes to rise the electron gas temperature and subsequently to heat the lattice itself [1-3]. From here on, all discussions correspond to the case when there is no laser-induced breakdown of the atmosphere - the effect of low-threshold air breakdown and its influence on the laser erosion of materials are discussed at length in Refs [13, 14]. The energy of electromagnetic radiation may be transferred into the metal by three main mechanisms of thermal conduction: the phonon, electron, and radiative ones. It is commonly supposed that phonon thermal conduction plays an appreciable role only at temperatures below $100 \mathrm{~K}$ and is therefore disregarded in laser plasma research. In the lattice temperature range of up to several thousand degrees the energy transfer mechanism is almost completely determined by electron thermal conduction. Lastly, radiative heat conductivity prevail when the temperature ranges from several thousand to tens of thousands degrees [1].
For a pulse duration $\tau \sim 10 \mathrm{~ns}$, the laser radiation energy dissipation in metals is qualitatively different both from the case of longer pulses $(\tau>1 \mu \mathrm{s})[1-5]$ and from the case of ultrashort ones $(\tau<100 \mathrm{ps})[15,16]$. This is due to the three main factors. First, the rise time of a nanosecond pulse $(\sim 1 \mathrm{~ns})$ approaches the characteristic excitation-energy relaxation time for the electron gas in a metal lattice (according to theoretical estimates, $\sim 10^{-10} \mathrm{~s}$ [1]), i.e. the rate of excitation transfer from the electron gas to the lattice is comparable to the input rate of the radiant energy which gives rise to this excitation. Greatly diminished in this case is the role of electron thermal conduction as the main mechanism of transferring into the target the energy absorbed by conduction electrons. Second, under high-intensity laser irradiation the reflectivity of a metal surface is not a constant value: beginning from some threshold intensity (according to different estimates, $I<$ $\left.100 \mathrm{MW} \mathrm{cm}^{-2}[1,17,18]\right)$, the initially high reflectivity (0.7-0.99 in the optical range, depending on the type of metal) may decrease several-fold during the irradiation, thereby lowering the absorptivity to $50 \%$ of the incident radiation energy [1]. Third, owing to a sharp lowering of the electrical conduction of metals observed in their rapid heating to temperatures of $1000-1500 \mathrm{~K}$, the depth of radiant energy penetration into the metals becomes several times longer. This fact impels us to reconsider the initial estimate of the depth of the metal surface layer in which the laser radiation interacts with conduction electrons: for laser irradiation of sufficiently high intensity it may range up not to $1 \mu \mathrm{m}$ but to $5 \mu \mathrm{m}$ and over [2].

The combined effect of these factors has the consequence that the disruption of metals by nanosecond laser pulses is hard to describe in the framework of the traditional thermal disruption model even for $I>10^{8} \mathrm{~W} \mathrm{~cm}^{-2}$. When the rise time of a laser pulse is sufficiently short $\left(10^{-8}-10^{-9} \mathrm{~s}\right)$, even for $I \sim$ $10^{8}-10^{9} \mathrm{~W} \mathrm{~cm}^{-2}$ the abrupt interphase boundary between the condensed metal phase and its vapour becomes nonabrupt and turns into a macroscopic transition layer, whose thickness is determined by the depth of light pulse penetration $(5-10 \mu \mathrm{m})$ [19]. In this layer there occurs dissipation of the laser pulse energy, resulting in its fast (as fast as permitted by the inertia of the matter) transformation to an overheated, rapidly expanding vapour (according to the estimates of Refs $[10,11]$, its initial expansion velocity amounts to $10-20 \mathrm{~km} \mathrm{~s}^{-1}$ ), which subsequently forms the erosion plume. This vigorous gas-dynamic process imparts a substantial recoil pressure momentum to the laser target, generating an acoustic shock wave propagating into the target material [20].

The combination of all the indicated processes eventually gives rise to a characteristic target surface relief (Figs $2 c-2 e$ ), which is significantly different from the relief produced under irradiation both by long [19] and ultrashort [15, 16] pulses. As is evident from the photographs, for a thermal disruption mechanism, which is typical for long-pulse irradiation, the resultant glassy target relief is a rather deep layer of solidified metal melt (Fig. 2b). 'Explosive' laser-produced erosion of the target surface gives rise to a characteristic microchannel structure with rather sharp edges. The roundings and roughness of the channel walls are estimated at a few micrometres. This is an indication that the liquid phase is practically missing from this laser irradiation regime (Figs 2c-2e). The combined effect of several factors (the initial target relief, the nonideality of target material, the nonuniformity of incident radiation intensity distribution in the 


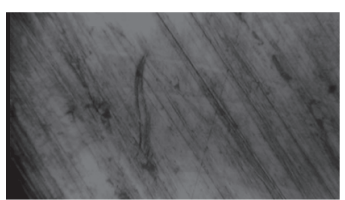

a

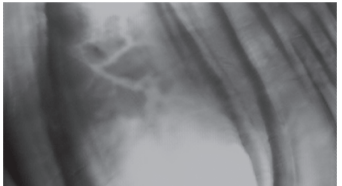

b

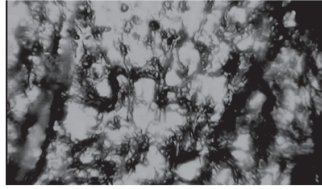

c

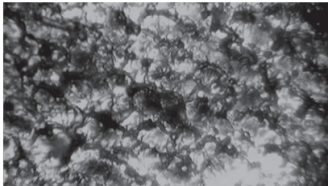

d

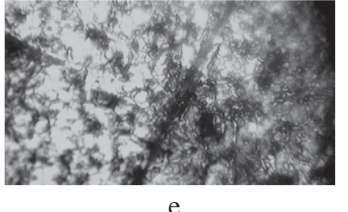

Figure 2. Photographs of target surface relief (a) prior to and (b) after irradiation by a long pulse of moderate intensity as well as (c, d, e) after irradiation of nickel, zinc, and lead, respectively, by a 100 -ns long pulse with an intensity of $10^{9} \mathrm{~W} \mathrm{~cm}^{-2}$. The images measure $150 \times 300 \mu \mathrm{m}$.

focal spot, the boundary gas-dynamic conditions, etc.) on the processes in the macrolayer gives rise to the recurrent pattern in the form of a microchannel network (possible for smooth and highly homogeneous targets is a pattern in the form of a microcrater set [21]).

With increasing power density of nanosecond pulses the microchannel structures become somewhat smoother due to the back action of the surface plasma on the target. This becomes possible due to the screening of the irradiation region by the laser-produced plasma and the complex nature of its interaction with the ambient gases (in a vacuum this effect is hardly pronounced [22]).

The detonation of a metal macrolayer, which possesses considerable excess energy, has the effect that there appears an intensely glowing plasma-vapour plume at the surface plasma region with a short delay relative to the onset of laser irradiation (5-10 ns for a pulse of duration $\tau=20 \mathrm{~ns}$ [11] and $30-40 \mathrm{~ns}$ for $\tau=100 \mathrm{~ns}$ [10]). This plume rapidly expands into the ambient medium and interacts with the irradiating laser pulse [12].

Experimental investigations into the gas-dynamic processes occurring in the laser erosion of metals by nanosecond pulses in the presence of rarefied gases or at atmospheric pressure [10-12] reveal qualitative difference in comparison with the vacuum case as regards the formation and glow of the vapour-plasma plume as well as its interaction with the incident radiation [23, 24]. As shown experimentally in Refs [10-12], the dynamics of integral plume glow under irradiation of metals ( $\mathrm{Zn}, \mathrm{Pb}, \mathrm{Ni}, \mathrm{Cu}, \mathrm{Ag}, \mathrm{Au}$ ) by 20- and 100ns long pulses (with their respective rise times of 5 and $30 \mathrm{~ns}$ ) for $I \sim 10^{8} \mathrm{~W} \mathrm{~cm}^{-2}$ reproduces the temporal pulse shape almost completely (with a short delay of $50 \%-60 \%$ of its duration). This testifies to the absence of significant interaction between the erosion plume and the incident radiation, which is additionally borne out by the data obtained by plume probing with auxiliary radiation at heights $h=1$ and $2 \mathrm{~mm}$ from the target surface: the vapour-plasma plume remains practically transparent for the probing radiation throughout its lifetime (only the plumes of fusible $\mathrm{Zn}$ and $\mathrm{Pb}$ represent a minor departure from this rule for 100-ns long pulses). A sharp increase in glow intensity as well as a significant lengthening of its descending part are observed on raising the power density of irradiating nanosecond pulses up to $10^{9} \mathrm{~W} \mathrm{~cm}^{-2}$. Simultaneously the plume transmittance to the probing radiation becomes lower (see Fig. 3); in this case, the probe radiation loss in the plume may be as high as $90 \%$ or more.

These facts suggest that the significant interaction between the erosion metal plume and the descending part of a nanosecond pulse begins when its intensity approaches $10^{9} \mathrm{~W} \mathrm{~cm}^{-2}$ (it is pertinent to note that this figure may somewhat vary with specific metal type due to the difference in thermophysical metal characteristics), the onset of appreciable radiation absorption by the plasma plume occurring considerably earlier than predicted by the thermal disruption model: even $40-50 \mathrm{~ns}$ after the onset of irradiation for $\tau=100 \mathrm{~ns}$ and $10 \mathrm{~ns}$ later for $\tau=20 \mathrm{~ns}$ instead of the 100-200 ns calculated [1].

The investigations of Refs $[11,12]$ showed that the peak of erosion plume glow for $\tau=20 \mathrm{~ns}\left(I \sim 10^{9} \mathrm{~W} \mathrm{~cm}^{-2}\right)$ is located at less than $1 \mathrm{~mm}$ from the target surface, while for pulses of similar intensity with $\tau=100 \mathrm{~ns}$ [10] at $1-2 \mathrm{~mm}$ (which is attributable to the higher energy density). This permits characterising the spatial scales of erosion plumes early in their nonstationary formation in atmospheric conditions. The data obtained in the probing of erosion plumes at 1 and $2 \mathrm{~mm}$ from
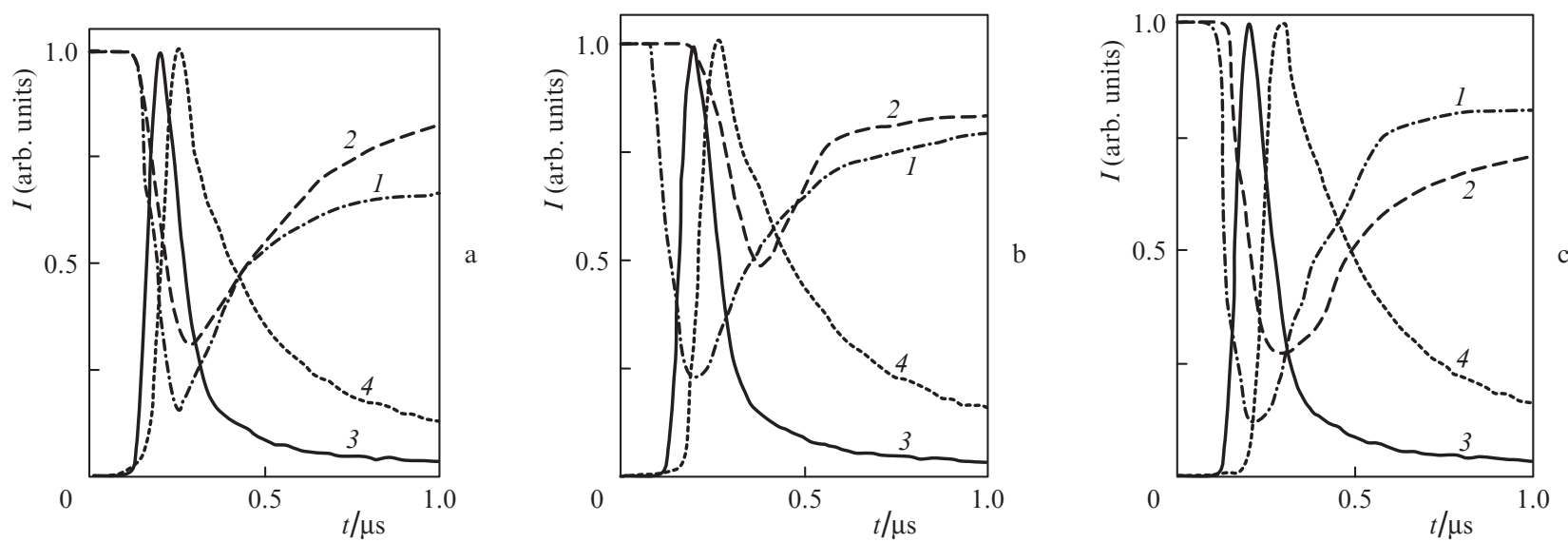

Figure 3. Time-dependent LPP transmittance for the probing radiation at (1) 1 and (2) 2 mm above the target for (a) zinc, (b) lead, and (c) nickel, as well as (3) irradiation pulse and (4) integral plume glow intensities as functions of time. The irradiation pulse duration is equal to 100 ns and the intensity is equal to $\sim 1 \mathrm{GW} \mathrm{cm}^{-2}$. 
$I$ (arb. units)

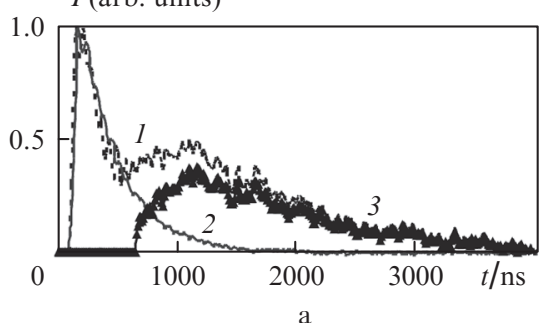

$I$ (arb. units)

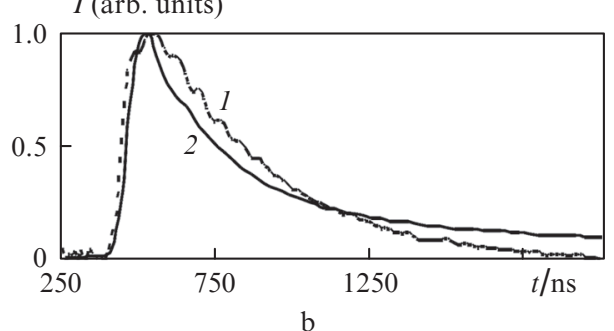

$I$ (arb. units)

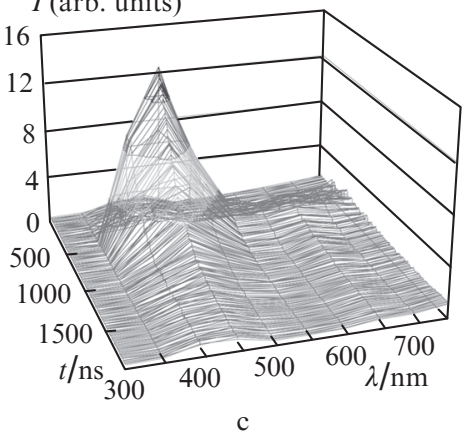

Figure 4. Dynamics of spectral luminosity under different conditions (see text)

the metal target surfaces permitted estimating the velocity of their propagation through these spaces at $4-15 \mathrm{~km} \mathrm{~s}^{-1}$ for $\tau=$ $20 \mathrm{~ns}$ [11] and at $7-20 \mathrm{~km} \mathrm{~s}^{-1}$ for $\tau=100 \mathrm{~ns}$ [10] $(I=$ $10^{9} \mathrm{~W} \mathrm{~cm}-2$ ), depending on the type of metal. The fact that shortening the leading edge of a pulse does not entail an increase in the initial velocity of the plasma plume suggests that these velocities are the limiting ones for the irradiation regime under discussion and that the initial plume dynamics is determined only by the macrolayer detonation velocity (i.e. by the amount of imparted excess energy).

Investigation of the spatiotemporal structure of erosion metal plumes $(\mathrm{Zn}, \mathrm{Pb}, \mathrm{Ni})$ for pulses with $\tau=20$ ns $(I \sim$ $10^{8}-10^{9} \mathrm{~W} \mathrm{~cm}^{-2}$ ) showed that the recorded time dependences of the glow in individual spectral intervals may be divided into two groups:

1. The plume glow in the spectral intervals corresponding to bright spectral lines of the metals. By way of example shown in Fig. 4a is the time dependence of the emission intensity in the spectral range corresponding to the characteristic line of $\mathrm{Pb}$ I lead atoms at a wavelength of $405.7 \mathrm{~nm}$ [curve (1)]. The emission in this range is due to the superposition of two processes: the continuous spectrum [curve (2)] is initially observed with a short ( $\sim 50 \%$ of the irradiating pulse duration) delay relative to the onset of irradiation, and then there appears, with a delay of $600-700$ ns relative to the onset of irradiation, the characteristic line itself, which emerges in the course of recombination of the corresponding ions [curve (3)].

2. The plasma plume glow in the spectral regions that do not contain the characteristic narrow lines of the metals. Given by way of example again is the corresponding dependence for the LPP of lead at a wavelength of $500.0 \mathrm{~nm}$ (Fig. 4b). One can see that by and large the emission dynamics in this spectral range reproduces the temporal shape of the integral plume emission and corresponds to the emission of the continuous spectrum.

Figure $4 \mathrm{c}$ shows the spectral-temporal shape of the emission of continuous spectrum. It has the shape of an asymmetric hill with a very steep front slope and a gently rear slope, with the emission peak at a wavelength of $\sim 450 \mathrm{~nm}$ reached approximately $40 \mathrm{~ns}$ after the onset of irradiation. In this case, the bulk of continuum emission is spatially confined within a height of $1 \mathrm{~mm}$ above the target.

According to the thermal model of laser-produced erosion, the picture of LPP emission must be qualitatively different: initially there is bound to be a burst of characteristic metal line emission arising from the sequential appearance of metal melt (formation of the melting front) and metal vapour (formation of vaporisation front) and the subsequent interac- tion of this vapour with the incident radiation. Next, due to the absorption of radiation energy the vapour heats up, ionises, and produces continuous plasma emission. For instance, according to the theoretical estimates of Ref. [7], when laser pulses with $\tau=15 \mathrm{~ns}$ irradiate aluminium with an intensity $I \sim$ $10^{9} \mathrm{~W} \mathrm{~cm}^{-2}$, a plasma plume begins to emerge with a delay of $\sim 15 \mathrm{~ns}$ relative to the onset of irradiation and becomes opaque (screens the target) 15 ns later. The duration of these processes (the LPP emergence and the attainment of opacity peak) agrees nicely with the data of the present work, but the succession of aggregate states in the irradiation region (solidfluid-vapour-plasma) is not confirmed by the data of spectral analysis. According to the thermal model, the plasma is heated during 15-30 ns after the onset of irradiation, and we should observe a different emission dynamics of characteristic lines: their spectral signal must appear 20-30 ns earlier than the continuous spectrum and the leading edge of their emission must therefore be longer. However, the perfect synchronism of these processes testifies to a different character of plasma production - to the detonation of the macrolayer, whereby the plasma is produced omitting intermediate aggregate states.

The resultant experimental information is of paramount importance in developing technologies for the laser deposition of metal nanostructures under atmospheric conditions [24-26], which is possible only in the $10^{8}-10^{9} \mathrm{~W} \mathrm{~cm}^{-2}$ range. Because, on the one hand, the liquid phase layer, which forms under the thermal character of disruption, is sputtered due to active gas-dynamic processes and gives rise primarily to large droplets (of diameter $10 \mu \mathrm{m}$ and over) [27] and, on the other hand, the strong laser radiation-plasma interaction in the hydrodynamic character of erosion drastically impairs the transfer of radiant energy to the target surface, thereby lowering the efficiency of action and, consequently, the removal of material.

\section{Conclusions}

According to experimental data, for the nanosecond range of laser pulses the transition from the quasi-stationary thermal mechanism of metal erosion to the explosive hydrodynamic one takes place when the irradiation power density is increased from $10^{8}$ to $10^{9} \mathrm{~W} \mathrm{~cm}{ }^{-2}$. This transition is characterised by a sharp strengthening of the interaction between the laser radiation and the vapour-plasma plume and the consequential lowering of its transmittance (to $10 \%$ and below) in a time corresponding to the duration of the leading edge of irradiating pulse, which is well below the estimates of the thermal 
erosion model. That this laser erosion mode corresponds to the hydrodynamic model is also borne out by the resultant relief of metal target surface, the independence of the initial velocity of the plasma-vapour plume on the steepness of the leading edge of the laser pulse, and the emission dynamics of the characteristic metal spectral lines.

Acknowledgements. The author expresses his deep gratitude to V.K. Goncharov for discussing the materials of the paper.

\section{References}

1. Anisimov S.I., Imas Ya.A., Romanov G.S., Khodyko Yu.V. Deistvie lazernogo izlucheniya bol'shoi moshchnosti na metally (Action of High-Power Laser Radiation on Metals) (Moscow: Nauka, 1970)

2. Mirkin L.I. Fizicheskie osnovy obrabotki materialov luchami lazera (Physical Foundations of Material Processing by Laser Beams) (Moscow: Izd. MGU, 1975).

3. Rykalin N.N., Uglov A.A., Kokora A.N. Lazernaya obrabotka materialov (Laser Processing of Materials) (Moscow: Mashinostroenie, 1975).

4. Prokhorov A.M., Konov V.I., Ursu I., Mikheilesku I.N. Vzaimodeistvie lazernogo izlucheniya s metallami (Interaction of Laser Radiation with Metals) (Moscow: Nauka, 1988).

5. Raizer Yu.P. Deistvie lazernogo izlucheniya (Action of Laser Radiation) (Moscow: Mir, 1974).

6. Anisimov S.I., Luk'yanchuk B.S. Usp. Fiz. Nauk, 172, 301 (2002) [Phys. Usp., 45, 293 (2002)].

7. Stankevich Yu.A., Stepanov K.L., Stanchits L.K., in 'Teplo- $i$ Massoperenos' (Thermal and Mass Transfer) (Minsk, izd. ITMO, 2007) p. 167.

8. Luk'yanchuk B.S., Marine W., Anisimov S.I., Simakina G.A. Proc. SPIE Int. Soc. Opt. Eng., 3618, 434 (1999).

9. Uglov A.A., Kokora A.N. Kvantovaya Elektron., 4 (6), 1189 (1977) [Sov. J. Quantum Electron., 7 (6) 671 (1977)]

10. Goncharov V.K., Kozadaev K.V., Shchegrikovich D.V. Inzh.-Fiz. Zh., 84, 723 (2011) [J. Eng. Phys. Thermophys., 84, 781 (2011)]

11. Goncharov V.K., Kozadaev K.V., Makarov V.V., Shchegrikovich D.V. Inzh.-Fiz. Zh., 86, 737 (2013) [J. Eng. Phys. Thermophys., 86, 798 (2013)].

12. Goncharov V.K., Kozadaev K.V., Shchegrikovich D.V. Zh. Prikl. Spektrosk., 80, 409 (2013) [J. Appl. Spectrosk., 80, 395 (2013)]

13. Kawahara N., Beduneau J.L., Nakayama T., Tomita E., Ikeda Y. Appl. Phys., 86, 605 (2011).

14. Klimentov S.M., Kononenko T.V., Pivovarov P.A., Konov V.I., Garnov S.V., et al. Trudy Inst. Obshchei Fiz. Ross. Akad. Nauk, 60, 13 (2004).

15. Anisimov S.I., Zhakhovskii V.V., Inogamov N.A., Nishihara K., Petrov Yu.V., Khokhlov V.A. Zh. Eksp. Teor. Fiz., 130, 2129 (2006).

16. Kononenko T.V., Konov V.I., Garnov S.V. et al. Kvantovaya Elektron., 28, 167 (1999) [Quantum Electron., 29, 724 (1999)].

17. Evtushenko A., Ivanik E., Rozhnyakovskii K. Inzh.-Fiz. Zh., 76, 10 (2003).

18. Banishev A.F., Balykina E.A. Kvantovaya Elektron., 24, 557 (1997) [ Quantum Electron., 27, 542 (1997)].

19. Kozadaev K.V. Perspektivnye Materialy, (6), 70 (2011).

20. Gus'kov K.S., Gus'kov S.Yu. Kvantovaya Elektron., 31, 305 (2001) [ Quantum Electron., 31, 305 (2001)].

21. Min'ko L.Y., Chivel Y.A. J. Phys. III, (4), 175 (1994).

22. Kubkowska M., Gasior P., Rosinski M., Wolowski J., Sadowski M.J., Malinowski K., Skladnik-Sadowska E. Eur. Phys. J., 54, 463 (2009).

23. Shuaibov A.K., Chuchman M.P., Shimon L.L. Tech. Phys. Lett., 30, 1042 (2004).

24. Goncharov V.K., Kozadaev K.V. Inzh.-Fiz. Zh., 83, 80 (2010) [J. Eng. Phys. Thermophys., 83, 90 (2010)].

25. Goncharov V.K., Kozadaev K.V., Shchegrikovich D.V. Inzh.-Fiz. Zh., 86, 754 (2013) [J. Eng. Phys. Thermophys., 86, 805 (2013)].

26. Goncharov V.K., Kozadaev K.V., Shchegrikovich D.V. Optical Memory Neural Networks (Information Optics), 20, 255 (2011).
27. Goncharov V.K. Inzh.-Fiz. Zh., 74, 87 (2001) [J. Eng. Phys. Thermophys., 74, 1173 (2001)]. 